\title{
Automatic tube potential selection with tube current modulation in coronary CT angiography: Can it achieve consistent image quality among various individuals?
}

\author{
XIAO-PING WANG* ${ }^{*}$ XIAO-MEI ZHU*, YIN-SU ZHU, WANG-YAN LIU, XIAO-HAN YANG, \\ WEI-WEI HUANG, YI XU and LI-JUN TANG
}

Department of Radiology, The First Affiliated Hospital of Nanjing Medical University, Nanjing, Jiangsu 210029, P.R. China

Received October 17, 2017; Accepted April 6, 2018

DOI: $10.3892 /$ etm.2018.6158

\begin{abstract}
The present study included a total of 111 consecutive patients who had undergone coronary computed tomography (CT) angiography, using a first-generation dual-source CT with automatic tube potential selection and tube current modulation. Body weight (BW) and body mass index (BMI) were recorded prior to $\mathrm{CT}$ examinations. Image noise and attenuation of the proximal ascending aorta (AA) and descending aorta (DA) at the middle level of the left ventricle were measured. Correlations between BW, BMI and objective image quality were evaluated using linear regression. In addition, two subgroups based on BMI (BMI $\leq 25$ and $>25 \mathrm{~kg} / \mathrm{m}^{2}$ ) were analyzed. Subjective image quality, image noise, the signal-to-noise ratio (SNR) and the contrast-to-noise ratio (CNR) were all compared between those. The image noise of the AA increased with the BW and BMI (BW: $r=0.453$, $\mathrm{P}<0.001$; BMI: $r=0.545, \mathrm{P}<0.001)$. The $\mathrm{CNR}$ and $\mathrm{SNR}$ of the AA were inversely correlated with BW and BMI, respectively. The image noise of the DA and the CNR and SNR of the DA exhibited a similar association to those with the BW or BMI. The $\mathrm{BMI}>25 \mathrm{~kg} / \mathrm{m}^{2}$ group had a significant increase in image noise $(33.1 \pm 6.9$ vs. $27.8 \pm 4.0 \mathrm{HU}, \mathrm{P}<0.05)$ and a significant reduction in CNR and SNR, when compared with those in the BMI $\leq 25 \mathrm{~kg} / \mathrm{m}^{2}$ group (CNR: $18.9 \pm 4.3$ vs. $16.1 \pm 3.7, \mathrm{P}<0.05$; SNR: $16.0 \pm 3.8$ vs. $13.6 \pm 3.2, \mathrm{P}<0.05)$. Patients with a BMI of $\leq 25 \mathrm{~kg} / \mathrm{m}^{2}$ had more coronary artery segments scored as excellent, compared with patients with a BMI of $>25 \mathrm{~kg} / \mathrm{m}^{2}$ $(\mathrm{P}=0.02)$. In conclusion, this method is not able to achieve a consistent objective image quality across the entire patient
\end{abstract}

Correspondence to: Dr Yi Xu or Dr Li-Jun Tang, Department of Radiology, The First Affiliated Hospital of Nanjing Medical University, 300 Guangzhou Road, Nanjing, Jiangsu 210029, P.R. China

E-mail: aleenxu@163.com

E-mail: 1034477400@qq.com

*Contributed equally

Key words: coronary computed tomography angiography, image noise, image consistency, body mass index, contrast-to-noise ratio population. The impact of BW and BMI on objective image quality was not completely eliminated. BMI-based adjustment of the tube potential may achieve a more consistent image quality compared to automatic tube potential selection, particularly in patients with a larger body habitus.

\section{Introduction}

Coronary CT angiography (CTA) has become an accurate and beneficial imaging modality for the non-invasive detection of coronary artery disease (CAD) $(1,2)$. With the increasing number of coronary CTAs performed, concerns regarding the exposure of patients to ionizing radiation have also increased (3). Numerous techniques to reduce the radiation dose by using hardware and certain acquisition protocols have been developed (4-10).

An automatic software algorithm, known as automatic tube potential selection with tube current modulation (APSCM; Siemens Healthcare, Munich, Germany), has been developed and used in coronary CTA. Tube potentials and currents are selected according to patients' anatomy and attenuation information from the initial CT topogram $(11,12)$. Based on the topographic image, the software calculates the total tissue attenuation along the $\mathrm{z}$-axis of the patient, and calculates the tube current that would be required for each of the tube potential settings on the basis of the user-defined examination type, image quality and noise preferences. The system then determines the optimal combination of tube potential and tube current to produce the desired image quality at the lowest dose per patient (in terms of volume CT dose index), and these settings are used to scan the patient. Lower tube potentials tend to apply in APSCM. A previous study reported that the use of APSCM for coronary CTA significantly reduced the radiation dose, compared with that applied in the method of tube potential modulation according to the body mass index (BMI), while maintaining the subjective image quality (13). However, a low tube voltage is accompanied with a higher image noise due to the lower X-ray beam penetration capability, particularly in obese patients. A persistent question is whether, after automatically selecting the tube potential and current, a consistent image quality may be achieved among various individuals. Thus far, only few studies have investigated the 
effect of this automatic software algorithm on the consistency of image quality (13).

The purpose of the present study was to investigate the influence of the body weight (BW) and BMI on the consistency of image quality in patients undergoing coronary CTA using the APSCM method.

\section{Materials and methods}

Patients. In February 2015, a total of 120 patients with suspected CAD who were referred to the Department of Radiology of the First Affiliated Hospital of Nanjing Medical University (Nanjing, China) for coronary CTA were enrolled in this prospective study. The indications were patients with chest pain suspected of having a low-to-intermediate risk of coronary lesions, which was in accordance with current guidelines and recommendations (2). Exclusion criteria were as follows: Impaired renal function ( $\mathrm{n}=3$ ), known hypersensitivity to iodinated contrast material $(n=2)$ and patients with arrhythmias $(n=4)$. A total of 111 patients (51 women and 60 men; mean age, $57.3 \pm 10.8$ years, median age, 57 years; range, $32-84$ years) with a mean BMI of $24.7 \pm 3.1 \mathrm{~kg} / \mathrm{m}^{2}$ (range, $17.9-36.9 \mathrm{~kg} / \mathrm{m}^{2}$ ) were enrolled in the present study. The institutional Review Board of the First Affiliated Hospital of Nanjing Medical University (Nanjing, China) approved the present study and informed consent was obtained from all patients.

Scan protocol. All examinations were performed using a first-generation Dual Source CT Scanner (Somatom ${ }^{\circledR}$ Definition; Siemens Healthcare). All CT scans were completed in a retrospective electrocardiogram-triggered manner. Scanning parameters were as follows: Detector collimation, $2 \times 32 \times 0.6 \mathrm{~mm}$; slice collimation, $2 \times 64 \times 0.6 \mathrm{~mm}$ by means of a z-flying focal spot; gantry rotation time, $330 \mathrm{msec}$; heart-rate adapted pitch, 0.20-0.42. $\beta$-receptor antagonists were administered when the heart rate was $>90 \mathrm{bpm}$. APSCM, a commercially available automated software algorithm (Care $\mathrm{kV}$ and CAREDose 4D; Siemens Healthcare), was applied for the selection of tube parameters. The Care $\mathrm{kV}$ type setting was maintained in the 'angiography' position for all patients indicating a robust performance for CTA. A slice thickness of $0.75 \mathrm{~mm}$ with an increment of $0.5 \mathrm{~mm}$ and medium soft-tissue reconstruction kernels by a filtered back projection (FBP) algorithm were used for the reconstruction of transverse images of the best diastolic and systolic phases.

High-concentration contrast material (Ultravist, iopromide 370 mg I/ml; Bayer Healthcare, Berlin, Germany) followed by saline was administered with a dual-head power injector (Dual Shot; Med Rad Inc., Indianola, PA, USA) via a 20-G needle, preferably inserted into the right cubital vein. The contrast material dosage during coronary CTA was calculated according to the BW and BMI using various coefficients, with the injection rate determined as the dosage divided by the scan time plus $8 \mathrm{sec}(14,15)$. For all patients, the scan delay times were determined by the test bolus technique with fixed tube parameters $(100 \mathrm{kV}, 45 \mathrm{mAs})$ and a fixed contrast protocol (20 $\mathrm{ml}$ contrast material at a flow rate of $4 \mathrm{ml} / \mathrm{sec}$ ).

Objective image quality. For objective image quality analysis, image noise and mean $\mathrm{CT}$ attenuations of the ascending aorta
(AA), descending aorta (DA) and proximal coronary arteries, including left main (LM), right coronary artery (RCA), left anterior descending artery (LAD) and left circumflex artery (LCX), were measured preferably at the best diastolic phase. The image noise was defined as the standard deviation of CT attenuation in a defined region of interest (ROI). ROIs of the AA were placed cranial to the left coronary ostium. ROIs of the DA were placed at the middle of the left ventricle. The ROIs were drawn to be as large as possible, which included $\sim 75 \%$ of lumen areas, with the exclusion of all plaques. To define the contrast-to-noise ratio (CNR), CT attenuation of the perivascular fat tissue was also measured. The signal-to-noise ratio (SNR) and CNR were calculated according to methods published in previous studies $(16,17)$.

Subjective image quality. Two experienced cardiovascular radiologists (one with 9 years and the other with 1 year of experience in coronary CTA) independently evaluated the subjective image quality of CTA images of coronary arteries. A total of 15 segments of coronary arteries were identified based on the established American Heart Association criteria (18). The subjective image quality was assessed by grading it on a 4-point Likert scale: 4, excellent image quality, defined as complete absence of motion artifacts, clear delineation of vessel wall with the ability to access luminal stenosis as well as plaque characteristics; 3, acceptable image quality, not compromising diagnostic vessel assessment; 2, reduced image quality due to motion, noise or low contrast, but still sufficient to rule out significant stenosis; 1 , non-diagnostic image quality (19). In cases of a lack of conformity between the two radiologists regarding the image assessment, consensus was reached by discussion.

Statistical analysis. Statistical analysis was performed using commercially available statistical software (SPSS v.13.0; SPSS, Inc., Chicago, IL, USA). Continuous variables are presented as the mean \pm standard deviation. A simple linear regression model was used to investigate the influences of $\mathrm{BW}$ and BMI on the image noise, CNR and SNR. All patients were divided into two subgroups based on their BMI (BMI $\leq 25$ or $>25 \mathrm{~kg} / \mathrm{m}^{2}$ ). Averages of the image noise, CNR and SNR of each of the two subgroups were compared using an independent Samples t-test. Nonparametric data were evaluated with the Mann-Whitney-U test. The inter-observer variability for subjective image quality scoring was determined by using linear weighted Cohen's kappa statistics. $\mathrm{P}<0.05$ was considered to indicate a statistically significant difference.

\section{Results}

Patient characteristics and scan parameters. A total of 111 patients (60 male, 51 female; age range, 32-84 years; mean age, 57.3 years; BMI range 17.9-36.9, mean BMI $24.7 \mathrm{~kg} / \mathrm{m}^{2}$ ) received CT examinations. One patient was selected $120 \mathrm{kV}$ using APSCM. Patient characteristics and coronary CTA parameters are summarized in Table I.

Objective image quality. Correlations between $B W$ and the image noise, SNR and CNR. A moderate positive correlation was identified for the BW against Noise $_{\mathrm{AA}}(\mathrm{r}=0.453, \mathrm{P}<0.001)$ and Noise $_{\mathrm{DA}}(\mathrm{r}=0.473, \mathrm{P}<0.001)$. In addition, a weakly positive correlation was noted between $\mathrm{BW}$ and the image noise of 
Table I. Patient characteristics and coronary CTA parameters.

\begin{tabular}{lc}
\hline Characteristic & Value \\
\hline Age (years) & $57.3 \pm 10.8$ \\
Male sex & $60(54.0)$ \\
Height $(\mathrm{cm})$ & $165 \pm 9$ \\
Body weight $(\mathrm{kg})$ & $67.9 \pm 11.3$ \\
BMI $\left(\mathrm{kg} / \mathrm{m}^{2}\right)$ & $24.7 \pm 3.1$ \\
Heart rate $($ beats/min) & $71.3 \pm 10.2$ \\
Tube potential $(\mathrm{kV})$ & \\
100 & $110(99.1)$ \\
120 & $1(0.9)$ \\
Tube current time $(\mathrm{mAs})$ & $228(180-315)$ \\
CTDI (mGy) & $25.7 \pm 4.8$ \\
DLP (mGy x cm) & $370.7 \pm 71.7$ \\
\hline
\end{tabular}

Values are expressed as the mean \pm standard deviation, median (range) or $\mathrm{n}(\%)$. BMI, body mass index; CTDI, computed tomography dose index; DLP, dose length product.

all coronary arteries, except for the RCA (Noise $_{\mathrm{LM}}: \mathrm{r}=0.279$, $\mathrm{P}=0.003$; Noise $_{\mathrm{LAD}}$ : $\mathrm{r}=0.288, \mathrm{P}=0.002$; Noise $_{\mathrm{LCX}}: \mathrm{r}=0.296$, $\mathrm{P}=0.002)$. A scattergram depicting the association between $\mathrm{BW}$ and Noise $_{\mathrm{DA}}$ is presented in Fig. 1. These results indicated that the image noise in the coronary CTA image increases with the BW when using the APSCM method. The CNR and SNR of the AA, DA, LM, LAD and LCX decreased with increasing $\mathrm{BW}$.

Correlation between BMI and image noise, CNR and SNR. A moderate, positive correlation was recorded between BMI and the image noise (Fig. 1), and moderate, negative correlations were identified between BMI, and the CNR and SNR (Fig. 2), indicating that the objective image quality was reduced in heavier patients. The coefficients for the associations between BW, BMI and image noise, SNR, CNR of the AA and DA from linear regression analysis are listed in Table II.

Comparison of image noise, CNR and SNR between groups subcategorized by BMI. Image noise, CNR and SNR of the AA and DA among all patients subcategorized by BMI were compared and are summarized in Table III and Fig. 3. A significant difference in image noise, SNR and CNR was identified between the two subgroups. For patients with a BMI of $>25 \mathrm{~kg} / \mathrm{m}^{2}$, the Noise $_{\mathrm{AA}}$ was higher than that for patients with a BMI of $\leq 25 \mathrm{~kg} / \mathrm{m}^{2}(33.1 \pm 6.9$ vs. $27.8 \pm 4.0 \mathrm{HU}, \mathrm{P}<0.05)$, but the CNR and SNR of the AA were lower (CNR: 16.1 \pm 3.7 vs. $18.9 \pm 4.3, \mathrm{P}<0.05$; SNR: $13.6 \pm 3.2$ vs. $16.0 \pm 3.8, \mathrm{P}<0.05)$. Similarly, the Noise $_{\mathrm{DA}}$ of the subgroup with a larger BMI was higher than that of the subgroup with a smaller BMI (35.9 \pm 6.1 vs. 29.8 $\pm 6.5 \mathrm{HU}, \mathrm{P}<0.05)$, while the $\mathrm{CNR}$ and $\mathrm{SNR}$ of the larger BMI subgroup were lower (CNR: $14.2 \pm 3.3$ vs. 17.2 \pm 4.1 , $\mathrm{P}<0.05$; SNR: $11.9 \pm 2.8$ vs. $14.4 \pm 3.5, \mathrm{P}<0.05)$.

Subjective image quality. A total of 1,415 coronary artery segments were evaluated. An inter-observer agreement regarding subjective image quality was substantial (kappa=0.761). The number of segments with scores of 2-4, considered to be diagnostic segments, was 1,413 (99.85\%). The subjective image quality was further compared in the two subgroups with different BMIs. Among the 777 segments of the smaller BMI group, 585 segments (75.29\%) were scored as 4, with excellent subjective image quality. Among the 638 segments in the larger BMI group, 453 segments $(71.00 \%)$ were scored as 4 . Patients with a BMI of $\leq 25 \mathrm{~kg} / \mathrm{m}^{2}$ had more coronary artery segments scored as excellent compared with patients with a BMI of $>25 \mathrm{~kg} / \mathrm{m}^{2}(\mathrm{P}=0.02$; Table IV).

\section{Discussion}

The present study indicated that the application of APSCM in coronary CTA maintains a subjective image quality, but that it cannot achieve uniform image quality among various individuals. The image noise, SNR and CNR were still correlated with the BW and BMI. Although the larger BMI group demonstrated a comparable subjective image quality to that in the smaller BMI group, the objective image quality was degraded.

APSCM assigns the optimal tube current and tube potential automatically for each patient depending on the type of study performed, the body region that is being imaged, and the patient's body habitus. It was considered that certain basic patient-specific information, including the BW and BMI, should be contributing factors in the selection of scanning parameters. In several studies, a lower tube potential (80 or $100 \mathrm{kV}$ ) was automatically selected for $>90 \%$ patients in the APSCM group. In addition, $80 \mathrm{kV}$ was selected in a small part of the cohort $(11,12)$. In the present study, $100 \mathrm{kV}$ was selected for all of the patients except one, although the BMI ranged from 17.9-36.9 kg/m ${ }^{2}$. Patients with a BMI of $\leq 25 \mathrm{~kg} / \mathrm{m}^{2}$ accounted for $\sim 55 \%$ of this consecutive cohort. The objective image quality, including image noise, SNR and CNR, was not consistent between individuals. The results indicated that low tube potentials tended to be more frequently selected with APSCM for radiation dose reduction. In general, the radiation dose is proportional to the tube current and the square of the tube potential. Consequently, lowering the tube voltage is one of the most effective dose reduction techniques. For coronary CTA scans, a low tube voltage increases the attenuation of iodinated contrast agents, which maintains the contrast between the vessel lumen and surrounding structures.

The complex associations among tube parameters, radiation dose and image quality render it problematic for radiographers to simultaneously achieve a consistent image quality between various individuals and the lowest possible dose of radiation. Various strategies have been implemented for coronary CTA in medical centers. Lu et al (20) demonstrated that measurement of the chest circumference may be used to adapt the tube voltage and current for individualized dose control with sustained diagnostic image quality. Suh et al (12) combined APSCM and sonogram-affirmed iterative reconstruction (IR) for coronary CTA. They noted that these methods significantly improve the objective image quality with a reduced radiation dose. However, they also demonstrated that no superior objective image quality could be achieved before IR was used. When the FBP algorithm was applied, increased image noise, 

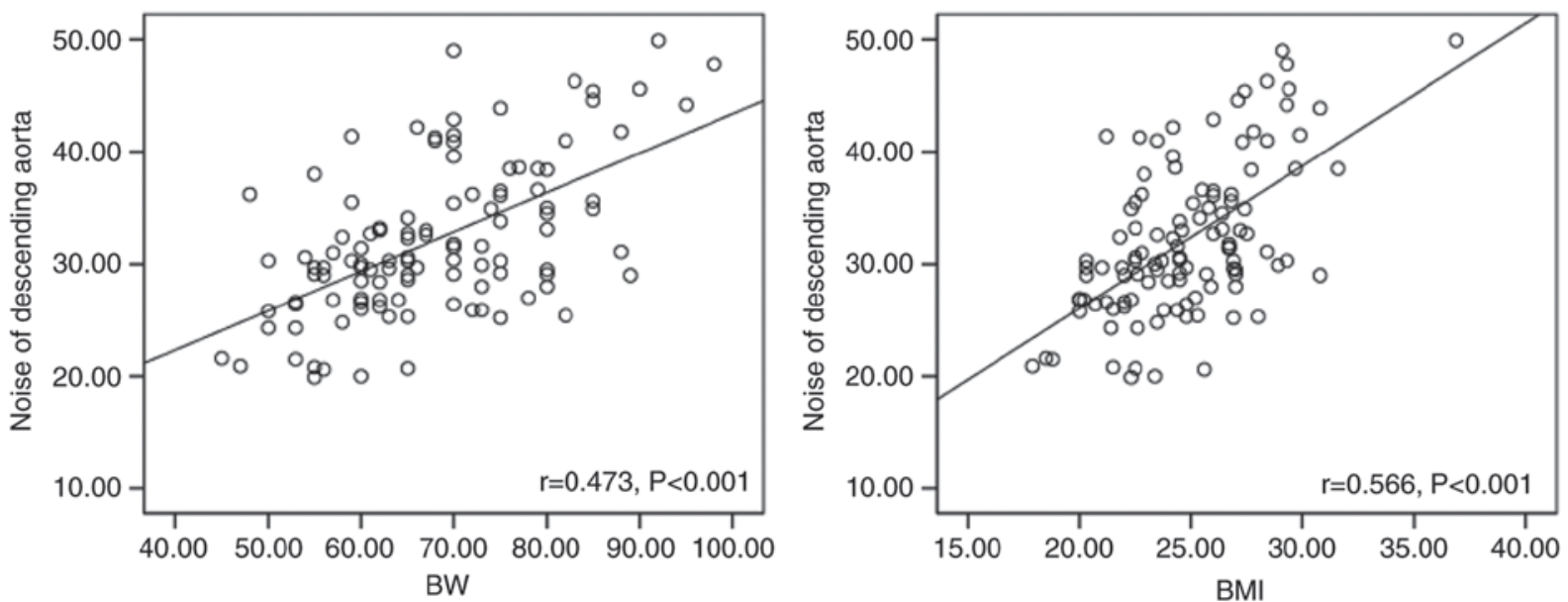

Figure 1. Correlation between BW, BMI and image noise of DA. The scattergrams depict a positive correlation between BW, BMI and image noise of DA. DA, descending aorta; BW, body weight; BMI, body mass index.
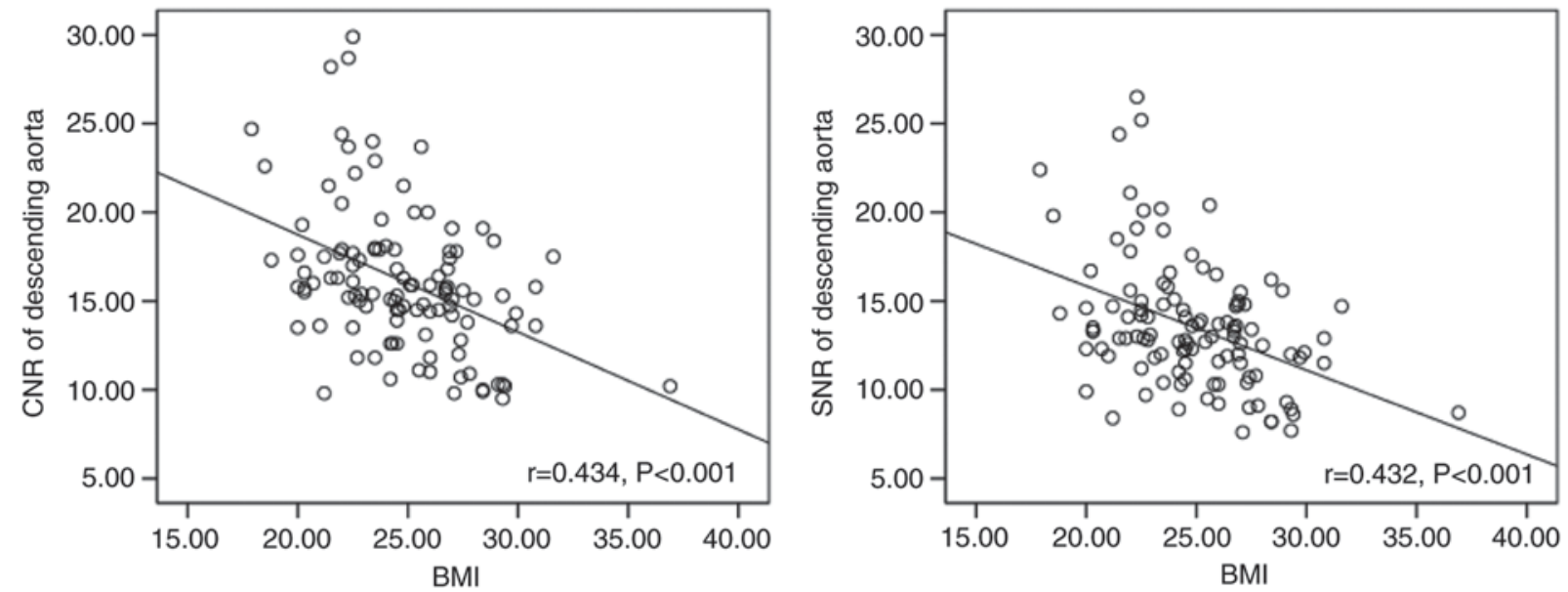

Figure 2. Correlation between BMI and CNR, SNR of the DA. The scattergrams depict a negative correlation between the BMI and the CNR or SNR of the DA. CNR, contrast-to-noise ratio; SNR, signal-to-noise ratio; DA, descending aorta; BMI, body mass index.
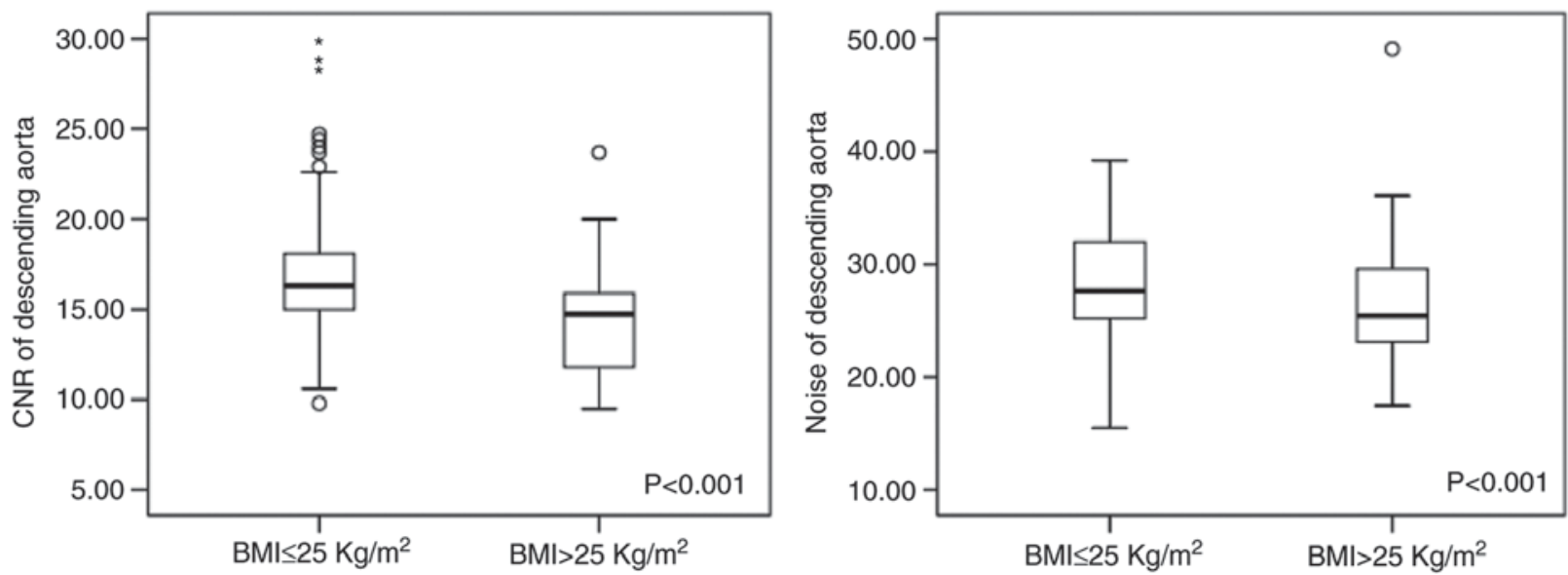

Figure 3. Comparison of noise and CNR of the descending aorta between patients with a BMI of $\leq 25 \mathrm{~kg} / \mathrm{m}^{2}$ and those with a BMI of $>25 \mathrm{~kg} / \mathrm{m}^{2}$. Horizontal lines represent the mean; boxes, meanquartile deviation; white dots, discrete values; and stars indicate extreme values. BMI, body mass index (calculated as weight in $\mathrm{kg} / \mathrm{height}$ in $\mathrm{m}^{2}$ ); $\mathrm{CNR}$, contrast-to-noise ratio.

and decreased CNR and SNR were observed in the APSCM group, compared with the standard dose coronary CTA.
In the subgroup analyses performed in the present study, it was also determined that the image noise in the BMI 
Table II. Statistical results on objective image quality.

\begin{tabular}{|c|c|c|c|c|c|}
\hline \multirow[b]{2}{*}{ Variable } & \multirow[b]{2}{*}{ Value } & \multicolumn{2}{|c|}{ BW } & \multicolumn{2}{|c|}{ BMI } \\
\hline & & $\mathrm{r}$ & $\mathrm{P}$-value & $\mathrm{r}$ & $\mathrm{P}$-value \\
\hline Noise of AA & $30.2 \pm 6.1$ & 0.453 & $<0.001$ & 0.545 & $<0.001$ \\
\hline CNR of AA & $17.6 \pm 4.3$ & 0.360 & $<0.001$ & 0.357 & $<0.001$ \\
\hline SNR of AA & $14.9 \pm 3.7$ & 0.362 & $<0.001$ & 0.352 & $<0.001$ \\
\hline Noise of DA & $32.5 \pm 7.0$ & 0.473 & $<0.001$ & 0.566 & $<0.001$ \\
\hline CNR of DA & $15.8 \pm 4.0$ & 0.439 & $<0.001$ & 0.434 & $<0.001$ \\
\hline SNR of DA & $13.3 \pm 3.4$ & 0.446 & $<0.001$ & 0.432 & $<0.001$ \\
\hline
\end{tabular}

Values are expressed as the mean \pm standard deviation. BMI, body mass index; CNR, contrast-to-noise ratio; SNR, signal-to-noise ratio; DA, descending aorta; AA, ascending aorta.

Table III. Noise, CNR and SNR of DA and AA in the two subgroups.

\begin{tabular}{lccc}
\hline Variable & $\mathrm{BMI} \leq 25 \mathrm{~kg} / \mathrm{m}^{2}$ & $\mathrm{BMI}>25 \mathrm{~kg} / \mathrm{m}^{2}$ & P-value \\
\hline Noise of AA & $27.8 \pm 4.0$ & $33.1 \pm 6.9$ & $<0.001$ \\
CNR of AA & $18.9 \pm 4.3$ & $16.1 \pm 3.7$ & $<0.001$ \\
SNR of AA & $16.0 \pm 3.8$ & $13.6 \pm 3.2$ & $<0.050$ \\
Noise of DA & $29.8 \pm 6.5$ & $35.9 \pm 6.1$ & $<0.001$ \\
CNR of DA & $17.2 \pm 4.1$ & $14.2 \pm 3.3$ & $<0.001$ \\
SNR of DA & $14.4 \pm 3.5$ & $11.9 \pm 2.8$ & $<0.001$
\end{tabular}

Values are expressed as the mean \pm standard deviation. BMI, body mass index; CNR, contrast-to-noise ratio; SNR, signal-to-noise ratio; DA, descending aorta; AA, ascending aorta.

$>25 \mathrm{~kg} / \mathrm{m}^{2}$ group was significantly higher than that in the $\mathrm{BMI} \leq 25 \mathrm{~kg} / \mathrm{m}^{2}$ group, while the CNR and SNR were lower. A previous study suggested that a higher tube current may be selected to overcome the increased image noise (21). However, a low $\mathrm{kV}$ cannot be used in all patients, as the tube current has limitations, and in patients with a high BMI and/or BW, the proper tube current cannot be emitted in a low-kV setting. In the present study, the objective image quality deteriorated in heavier patients. Numerous studies have indicated that advanced modeled iterative reconstruction may be used to decrease image noise $(22,23)$. IR entails fewer assumptions regarding noise distribution with an image, and operates with an iterative process of mathematical and statistical modeling to reduce image noise. When image noise is increased in the low-kV potential, IR is an effective method to compensate for image impairment. According to our experience, the use of APSCM combined with IR for coronary CTA allows for a reduction in radiation dose whilst maintaining the image quality. However, in the present study, only the FBP algorithm with the first-generation Dual Source CT Scanner was used.

The optimal image noise, SNR and CNR for diagnostic accuracy in coronary CTA were not fully determined in the present study. Different levels of image noise have been reported with distinct protocols. Shen et al (24) maintained the
Table IV. Subjective image quality between the two subgroups.

\begin{tabular}{lccc}
$\begin{array}{l}\text { Image quality } \\
\text { score }\end{array}$ & $\mathrm{BMI} \leq 25 \mathrm{~kg} / \mathrm{m}^{2}$ & $\mathrm{BMI}>25 \mathrm{~kg} / \mathrm{m}^{2}$ & P-value \\
\hline $1-3$ & $192(24.7)$ & $185(29.0)$ & 0.02 \\
4 & $585(75.3)$ & $453(71.0)$ &
\end{tabular}

Values are expressed as $\mathrm{n}_{\text {segments }}(\%)$. BMI, body mass index.

image noise at the target value of $35 \mathrm{HU}$ using the noise-based tube current reduction method with IR; the SNR of the image was $\sim 12$. According to the subjective 4-point scale evaluation, all images were accepted for diagnosis in their study. In another study, an image with a higher image noise and SNR (image noise, $40 \mathrm{HU}$; SNR, 14) in the APSCM group was also accepted as being of diagnostic image quality (12). In the present study, the image noise of the AA was $33.1 \pm 6.9$ and $27.8 \pm 4.0 \mathrm{HU}$ in the $\mathrm{BMI}>25 \mathrm{~kg} / \mathrm{m}^{2}$ and $\mathrm{BMI} \leq 25 \mathrm{~kg} / \mathrm{m}^{2}$ subgroups, respectively, while the subjective image quality was not significantly different between these two subgroups. One possible explanation was that the subjective image quality was affected by several factors, including heart rate, image noise, contrast attenuation and calcification on the vessel walls.

Subjective image quality was assessed according to the physicians' experience and knowledge in coronary CTA. The absolute CNR and SNR are sufficiently high in coronary CTA, and thus, increased image noise does not affect the subjective judgment of images from the larger BMI subgroup. Although the subjective image quality of the majority of arteries met the requirement for clinical diagnosis in the present study, it was considered that the deteriorated objective image quality in the larger BMI subgroup may affect the detection of non-calcified plaques, particularly by less experienced physicians.

The present study had several limitations. First, the diagnostic accuracy was not evaluated, as only a small number of patients underwent invasive coronary angiography. Furthermore, instead of IR, only the FBP algorithm was applied due to the scanner's limitation. As is known, more advanced iterative techniques further improve the image quality with substantially decreased 
image noise. In addition, the objective image quality was evaluated for coronary arteries and aorta; however, the objective image quality of coronary arteries was slightly or not linearly correlated with the BW and BMI. It appears that the objective image quality of the aorta is better than that of parameters of coronary arteries due to the deviation arising from measurement. Finally, the software was only applied in a relatively small number of patients, and $100 \mathrm{kV}$ was selected in the majority of them. Therefore, the objective parameters cannot be entirely matched to all possible determinants in different individuals.

In conclusion, the APSCM method in coronary CTA is not able to achieve consistent objective image quality across the entire patient population. The impact of the BW and BMI on objective image quality was not completely eliminated by APSCM. Greater image noise, and reduced CNR and SNR were recorded in patients with larger BMIs. Clinically, BMI-based adjustment of the tube potential may achieve a better, consistent image quality compared to automatic tube potential selection, particularly in patients with a larger body habitus. Combined with IR, this may resolve the inconsistency in objective image quality of coronary CTA among various individuals to a certain extent.

\section{Acknowledgements}

Not applicable.

\section{Funding}

No funding received.

\section{Availability of data and materials}

The datasets used and/or analyzed during the current study are available from the corresponding author on reasonable request.

\section{Authors' contributions}

XPW analyzed the patient data and drafted the manuscript. XMZ conceived and designed the study. XPW wrote the manuscript. YSZ interpreted the patient data. WYL and WWH evaluated the coronary scores. XHY contributed to data analysis. YX helped perform the analysis with constructive discussion. LJT helped interpret the results and played an important role in revising manuscript.

The final version of the manuscript has been read and approved by all authors, and each author believes that the manuscript represents honest work.

\section{Ethical approval and consent to participate}

The institutional Review Board of the First Affiliated Hospital of Nanjing Medical University (Nanjing, China) approved the present study and informed consent was obtained from all patients.

\section{Consent for publication}

Not applicable.

\section{Competing interests}

The authors declare that they have no competing interests.

\section{References}

1. Xu Yi, Tang L, Zhu X, Xu H, Tang J, Yang Z, Wang L and Wang D: Comparison of dual-source ct coronary angiography and conventional coronary angiography for detecting coronary artery disease. Int J Cardiovasc Imag 26 (Suppl 1): S75-S81, 2010.

2. Kim YJ, Yong HS, Kim SM, Kim JA, Yang DH and Hong YJ; Korean Society of Radiology; Korean Society of Cardiology: Korean guidelines for the appropriate use of cardiac CT. Korean J Radiol 16: 251-285, 2015.

3. Delewi R, Hoebers LP, Råmunddal T, Henriques JP, Angerås $\mathrm{O}$, Stewart J, Robertsson L, Wahlin M, Petursson P, Piek JJ, et al: Clinical and procedural characteristics associated with higher radiation exposure during percutaneous coronary interventions and coronary angiography. Circ Cardiovasc Interv 6: 501-506, 2013.

4. Meyersohn NM, Szilveszter B, Staziaki PV, Scholtz JE, Takx RAP, Hoffmann U and Ghoshhajra BB: Coronary CT angiography in the emergency department utilizing second and third generation dual source CT. J Cardiovasc Comput Tomogr 11: 249-257, 2017.

5. Halliburton SS, Abbara S, Chen MY, Gentry R, Mahesh M, Raff GL, Shaw LJ and Hausleiter J; Society of Cardiovascular Computed Tomography: SCCT guidelines on radiation dose and dose-optimization strategies in cardiovascular CT. J Cardiovasc Computed Tomography 5: 198-224, 2011.

6. He G, Liu X, Liu Y, Wang W and Ke Z: Dose study of electrocardiogram automatic tube current modulation technology in prospective coronary computed tomography angiography scans of overweight patients. Exp Ther Med 9: 2384-2388, 2015.

7. LaBounty TM, Leipsic J, Poulter R, Wood D, Johnson M, Srichai MB, Cury RC, Heilbron B, Hague C, Lin FY, et al: Coronary $\mathrm{CT}$ angiography of patients with a normal body mass index using $80 \mathrm{kVp}$ versus $100 \mathrm{kVp}$ : A prospective, multicenter, multivendor randomized trial. AJR Am J Roentgenol 197: W860-W867, 2011.

8. Wenz H, Maros ME, Meyer M, Gawlitza J, Förster A, Haubenreisser H, Kurth S, Schoenberg SO, Groden C and Henzler T: Intra-individual diagnostic image quality and organ-specific-radiation dose comparison between spiral cCT with iterative image reconstruction and z-axis automated tube current modulation and sequential cCT. Eur J Radiol Open 3: 182-190, 2016.

9. Scholtz JE, Wichmann JL, Hüsers K, Beeres M, Nour-Eldin NE, Frellesen C, Vogl TJ and Lehnert T: Automated tube voltage adaptation in combination with advanced modeled iterative reconstruction in thoracoabdominal third-generation 192-slice dual-source computed tomography: Effects on image quality and radiation dose. Acad Radiol 22: 1081-1087, 2015.

10. Ghoshhajra BB, Engel LC, Károlyi M, Sidhu MS, Wai B, Barreto M, Shanmugam U, Hoffmann U, Brady TJ, Kalra M and Abbara S: Cardiac computed tomography angiography with automatic tube potential selection: Effects on radiation dose and image quality. J Thorac Imaging 28: 40-48, 2013.

11. Layritz C, Muschiol G, Flohr T, Bietau C, Marwan M, Schuhbaeck A, Schmid J, Ropers D, Achenbach S and Pflederer T: Automated attenuation-based selection of tube voltage and tube current for coronary CT angiography: Reduction of radiation exposure versus a BMI-based strategy with an expert investigator. J Cardiovasc Comput Tomogr 7: 303-310, 2013.

12. Suh YJ, Kim YJ, Hong SR, Hong YJ, Lee HJ, Hur J and Choi BW: Combined use of automatic tube potential selection with tube current modulation and iterative reconstruction technique in coronary CT angiography. Radiolog 269: 722-729, 2013.

13. Park YJ, Kim YJ, Lee JW, Kim HY, Hong YJ, Lee HJ, Hur J, Nam JE and Choi BW: Automatic Tube Potential Selection with Tube Current Modulation (APSCM) in coronary CT angiography: Comparison of image quality and radiation dose with conventional body mass index-based protocol. J Cardiovasc Comput 6: 184-190, 2012.

14. Zhu X, Shi Z, Zhu Y, Liu W, Yang G, Yu T, Tang L and Xu Y: Individually adapted tube current selection and contrast medium injection protocol of coronary CT angiography based on test bolus parameters: A feasibility study. Acta Radiol 56: 666-672, 2015. 
15. Zhu X, Zhu Y, Xu H, Wan Y, Choo KS, Yang G, Tang L and $\mathrm{Xu}$ Y: An individualized contrast material injection protocol with respect to patient-related factors for dual-source CT coronary angiography. Clin Radiol 69: e86-e92, 2014.

16. Lembcke A, Wiese TH, Schnorr J, Wagner S, Mews J, Kroencke TJ, Enzweiler CN, Hamm B and Taupitz M: Image quality of noninvasive coronary angiography using multislice spiral computed tomography and electron-beam computed tomography. Invest Radiol 39: 357-364, 2004.

17. Ferencik M, Nomura $\mathrm{CH}$, Maurovich-Horvat $\mathrm{P}$, Hoffmann U, Pena AJ, Cury RC, Abbara S, Nieman K, Fatima U, Achenbach S and Brady TJ: Quantitative parameters of image quality in 64-slice computed tomography angiography of the coronary arteries. Eur J Radiol 57: 373-379, 2006.

18. Austen WG, Edwards JE, Frye RL, Gensini GG, Gott VL, Griffith LS, McGoon DC, Murphy ML and Roe BB: A reporting system on patients evaluated for coronary artery disease. Report of the Ad Hoc Committee for Grading of Coronary Artery Disease, Council on Cardiovascular Surgery, American Heart Association. Circulation 51(Suppl 4): S5-S40, 1975.

19. Lee AM, Beaudoin J, Engel LC, Sidhu MS, Abbara S, Brady TJ, Hoffmann U and Ghoshhajra BB: Assessment of image quality and radiation dose of prospectively ECG-triggered adaptive dual-source coronary computed tomography angiography (cCTA) with arrhythmia rejection algorithm in systole versus diastole: A retrospective cohort study. Int J Cardiovasc Imaging 29: 1361-1370, 2013.

20. Lu C, Wang Z, Ji J, Wang H, Hu X and Chen C: Evaluation of a chest circumference-adapted protocol for low-dose 128-slice coronary CT angiography with prospective electrocardiogram triggering. Korean J Radiol 16: 13-20, 2015.
21. Hausleiter J, Martinoff S, Hadamitzky M, Martuscelli E, Pschierer I, Feuchtner GM, Catalán-Sanz P, Czermak B, Meyer TS, Hein F, et al: Image quality and radiation exposure with a low tube voltage protocol for coronary $\mathrm{CT}$ angiography results of the PROTECTION II Trial. JACC Cardiovasc Imaging 3: 1113-1123, 2010.

22. Gordic S, Desbiolles L, Sedlmair M, Manka R, Plass A, Schmidt B, Husarik DB, Maisano F, Wildermuth S and Alkadhi H: Optimizing radiation dose by using advanced modelled iterative reconstruction in high-pitch coronary CT angiography. Eur Radiol 26: 459-468, 2016.

23. Abdullah KA, McEntee MF, Reed W and Kench PL: Radiation dose and diagnostic image quality associated with iterative reconstruction in coronary $\mathrm{CT}$ angiography: A systematic review. J Med Imaging Radiat Oncol 60: 459-468, 2016.

24. Shen J, Du X, Guo D, Cao L, Gao Y, Yang Q, Li P, Liu J and Li K: Prospective ECG-triggered coronary CT angiography: Clinical value of noise-based tube current reduction method with iterative reconstruction. PLoS One 8: e65025, 2013.

This work is licensed under a Creative Commons Attribution-NonCommercial-NoDerivatives 4.0 International (CC BY-NC-ND 4.0) License. 